\title{
Growth, structural and optical properties of novel nonlinear optical potassium phthalate di lithium borate (KPDLiB) single crystals
}

\author{
K. Mohanraj ${ }^{1,2}$, D. Balasubramanian ${ }^{1, *}$, N. JhANSi ${ }^{1}$ \\ ${ }^{1}$ Raman Research Laboratory, PG \& Research Department of Physics, Government Arts College, Tiruvannamalai-606603, \\ Tamilnadu, India \\ ${ }^{2}$ Department of Environmental Engineering and Management, Chaoyang University of Technology, Taichung 41349, Taiwan
}

\begin{abstract}
Novel nonlinear optical semi-organic, potassium phthalate di lithium borate (KPDLiB) single crystals were successfully grown by the slow solvent evaporation technique. Good crystalline nature and an orthorhombic structure were confirmed by powder X-ray diffraction and single crystal X-ray diffraction studies. The functional groups of KPDLiB were identified using FT-IR spectrum recorded in the range of $4000 \mathrm{~cm}^{-1}$ to $450 \mathrm{~cm}^{-1}$. UV-Vis spectrum showed transmitting ability of the crystals in the entire visible region. The photoluminescence spectrum exhibited good fluorescence emission in a visible region at $384 \mathrm{~nm}, 416 \mathrm{~nm}$ and $578 \mathrm{~nm}$. The second harmonic generation efficiency of the grown crystal was evaluated from Kurtz powder technique.
\end{abstract}

Keywords: X-ray diffraction; photoluminescence; nonlinear optical materials

\section{Introduction}

Nonlinear optical materials play an important role in optical signal processing, optical data storage, frequency conversion, telecommunications and optical information technology. The organic crystals have high nonlinear susceptibility, low thermal stability, poor mechanical strength and inorganic crystals have a good mechanical strength, high thermal stability and poor optical nonlinearity. The semi-organic crystals possess high nonlinearity, high mechanical strength, good optical susceptibility, ultra-fast response time and high optical damage threshold. The alkali metal ions have higher charge density in comparison to other metal ions and the ability to combine with organic and inorganic complexes [1-6]. The phthalate and borate family complexes have excellent properties for ultraviolet nonlinear optics. They are widely used in electronic and optoelectronic applications [7, 8]. KAP is an interesting material as it is used as the analyzer in X-ray spectroscopy $[9,10]$.

*E-mail: balad67@ rediffmail.com
It is a semi-organic compound with orthorhombic crystalline structure with space group Pca2 $2_{1}$. Its electrical, optical and nonlinear properties have been studied [11, 12].

The synthesis, growth, linear and nonlinear optical properties of the novel semi-organic crystals KPDLiB have been reported in this paper for the first time. The KPDLiB has a good linear and nonlinear properties, it can be used in optoelectronic applications.

\section{Experimental}

\subsection{Material synthesis}

High (99.9 \%) purity Merck grade potassium hydrogen phthalate, boric acid and lithium carbonate were taken in equimolar ratio and dissolved in deionized water and stirred by a magnetic stirrer for more than 4 hours at a temperature slightly higher than the room temperature. The prepared solution was allowed to dry at room temperature. The KPDLiB reaction scheme is shown in Scheme 1. Care was taken during heating of the solution 
and a maximum temperature of $50{ }^{\circ} \mathrm{C}$ was maintained in order to avoid its decomposition. The synthesized KPDLiB salt was dissolved in Millipore water and the solution was prepared in slightly undersaturated condition. The solution was constantly stirred for 8 hours using magnetic stirrer and then filtered using 4-micrometer filter by a peristaltic pump. Then the filtered solution was poured into different Petri dishes and covered by porous papers. The seed crystals were grown over a period of one week. Good quality seed crystals were used for the growth of large size crystals.

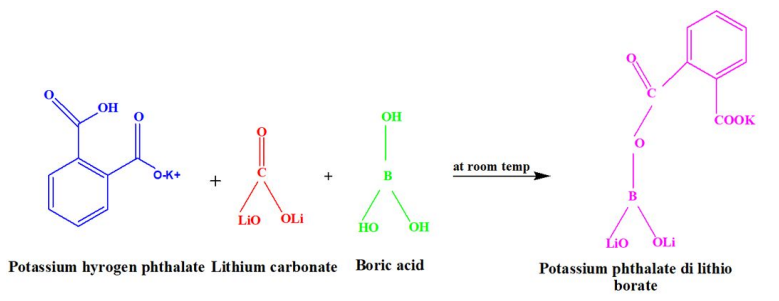

Scheme 1. Scheme for synthesis of KPDLiB.

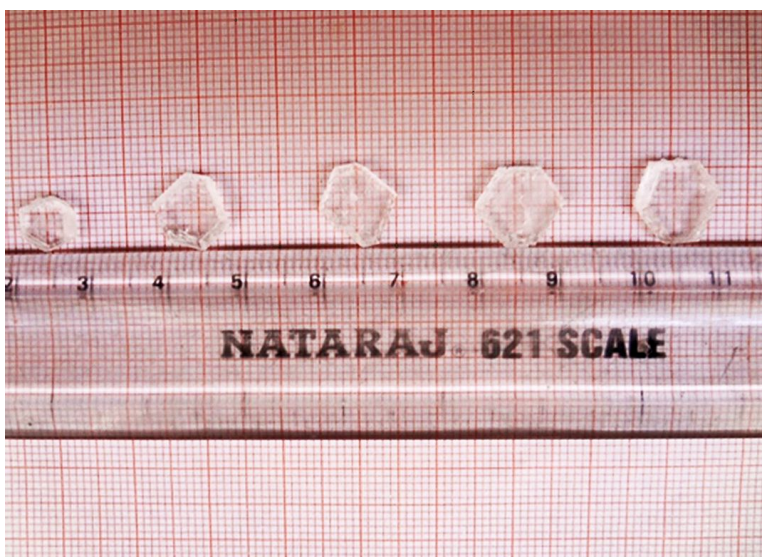

Fig. 1. KPDLiB single crystals grown by slow evaporation technique.

The saturated KPDLiB solution $(250 \mathrm{~mL})$ was prepared at room temperature and then filtered. In order to obtain supersaturation, the prepared solution was kept for two days to evaporate. One of the best seeds obtained was tied hung in the supersaturated solution. After introducing the seed crystal, KPDLiB single crystal was grown to the considerable size in a period of twenty days. The KPDLiB single crystals grown in various attempts are shown in Fig. 1.

\section{Results and discussion}

A finely crushed powder of KPDLiB crystal was subjected to powder X-ray diffraction analysis using a Rich Seifert diffractometer with $\mathrm{CuK} \alpha$ $(\lambda=1.5418 \AA)$ radiation. The sample was scanned over the range of $10^{\circ}$ to $80^{\circ}$ at a scan rate of $2 \% \mathrm{~min}$. The recorded X-ray pattern of KPDLiB is shown in Fig. 2, it sharp peaks show the good crystalline nature of the grown crystal.

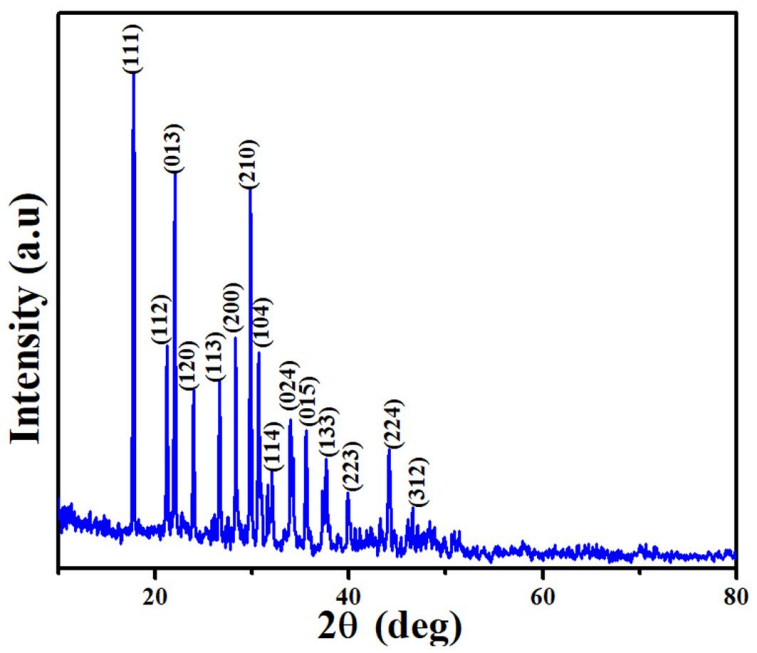

Fig. 2. The powder X-ray diffraction pattern of KPDLiB crystal.

The KPDLiB crystals were subjected to single crystal X-ray diffraction analysis using a Bruker Kappa APEX-2 diffractometer with $\operatorname{MoK} \alpha(\lambda=$ $0.7170 \AA$ ) radiation. The lattice parameters are listed in Table 1. It is found that the grown crystal belongs to an orthorhombic system with the space group $\mathrm{P} 2{ }_{1} 2{ }_{1} 2{ }_{1}$, a non-centrosymmetric system which satisfies the essential requirement for nonlinear optical properties.

In order to qualitatively analyze the presence of functional groups in the grown NLO single crystal, the FT-IR spectrum was recorded between $400 \mathrm{~cm}^{-1}$ and $4000 \mathrm{~cm}^{-1}$ using IFS BRUKER $66 \mathrm{~V}$ spectrometer by $\mathrm{KBr}$ pellet technique and the resultant spectrum is shown in Fig. 3. The 
Table 1. Single crystal XRD data of KPDLiB crystal.

\begin{tabular}{cccccccc}
\hline Sample & \multicolumn{6}{c}{ Lattice parameters } \\
\hline \multirow{3}{*}{ KPDLiB } & $\mathrm{a}$ & $\mathrm{b}$ & \multicolumn{8}{c}{$\alpha$} & $\alpha$ & $\beta$ & $\gamma$ & $\mathrm{V}$ \\
& {$[\AA]$} & {$[\AA ⿻$} & $\mathrm{c}[\AA]$ & {$\left[{ }^{\circ}\right]$} & {$\left[^{\circ}\right]$} & $\left.{ }^{\circ}\right]$ & {$\left[\AA^{3}\right]$} \\
\cline { 2 - 8 } & 6.35 & 9.44 & 13.10 & 90 & 90 & 90 & 785 \\
\hline
\end{tabular}

broad band appearing at $2792 \mathrm{~cm}^{-1}$ is due to $\mathrm{H}-\mathrm{C}=\mathrm{O}$; C-H stretching, the peaks at $2634 \mathrm{~cm}^{-1}$ and $2458 \mathrm{~cm}^{-1}$ indicate the presence of $\mathrm{O}-\mathrm{H}$ stretching. The carbonyl stretching $\mathrm{C}=\mathrm{O}$ is found to occur near $1666 \mathrm{~cm}^{-1}$. A sharp band observed at $1556 \mathrm{~cm}^{-1}$ and $1481 \mathrm{~cm}^{-1}$ has been assigned to the $\mathrm{C}-\mathrm{C}$ stretch (in ring). The $\mathrm{B}-\mathrm{O}$ symmetric stretching appears at $1257 \mathrm{~cm}^{-1}$ and $762 \mathrm{~cm}^{-1}$ with very strong intensity [13]. The small sharp peaks observed at $1146 \mathrm{~cm}^{-1}$ and $1083 \mathrm{~cm}^{-1}$ have been assigned to $\mathrm{C}-\mathrm{O}$ stretching. The $-\mathrm{C} \equiv \mathrm{C}-\mathrm{H}$ and $\mathrm{C}-\mathrm{H}$ bending is indicated by the peaks at $683 \mathrm{~cm}^{-1}$ and $647 \mathrm{~cm}^{-1}$. The alkali metal ion vibration is observed at $549 \mathrm{~cm}^{-1}$ [14]. All these assignments confirm the presence of potassium and borate ions in the crystal lattice.

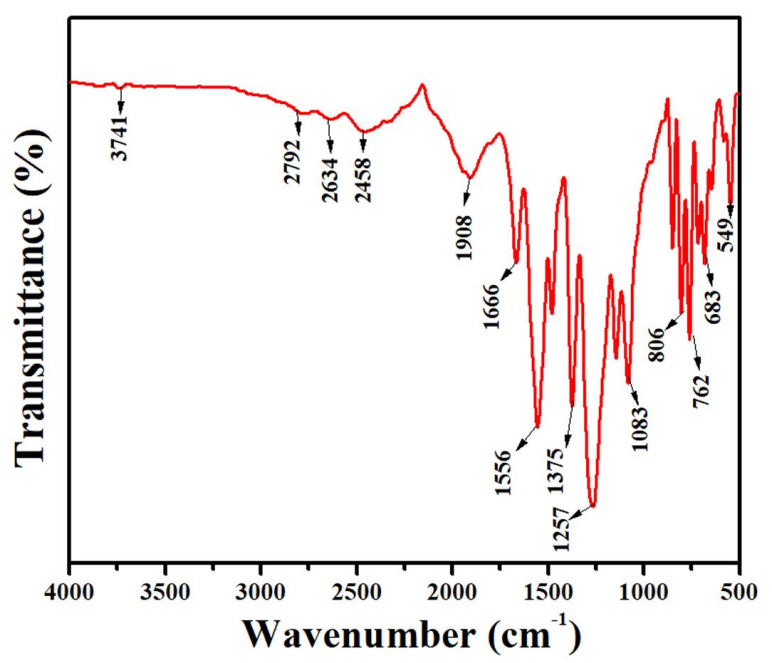

Fig. 3. FT-IR of spectrum of KPDLiB single crystal

The optical transparency of $2 \mathrm{~mm}$ thick KPDLiB single crystal was recorded using a PerkinElmer Lambda 35 UV-Vis spectrometer in the range of $200 \mathrm{~nm}$ to $900 \mathrm{~nm}$. The plot of percentage transmittance vs. wavelength $(\mathrm{nm})$ is shown in Fig. 4. The lower cut-off wavelength of the
KPDLiB crystal appearing at $327 \mathrm{~nm}$ is due to the $\pi \rightarrow \pi^{*}$ transition. The crystal has around $60 \%$ transmission in the entire visible region. The good transparent window at the wide wavelength range makes the grown crystals suitable for optical devices applications $[15,16]$.

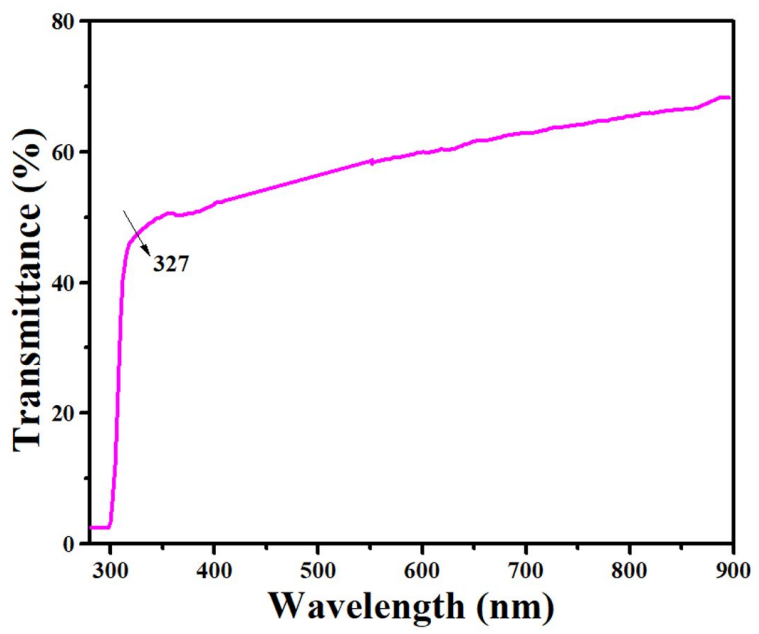

Fig. 4. UV- Vis spectrum of KPDLiB single crystal.

Photoluminescence is the process of emission of light when the atoms are excited from the ground state. The photoluminescence (PL) spectrum recorded between $300 \mathrm{~nm}$ and $650 \mathrm{~nm}$ is depicted in Fig. 5 with an excitation wavelength of $327 \mathrm{~nm}$. The recorded spectrum shows the broad emission in the ultraviolet range observed at $384 \mathrm{~nm}$. The emission peaks occuring at $416 \mathrm{~nm}$ and $578 \mathrm{~nm}$ indicate the violet and green emissions which are suitable for photonic device applications [17, 18].

The SHG properties of KPDLiB crystal were studied using Kurtz powder test. The grown single crystals of KPDLiB and reference samples (KDP and urea) were converted into uniform particles of $125 \mu \mathrm{m}$ to $150 \mu \mathrm{m}$ in size and packed into a microcapillary tube. The fundamental beam of an Nd:YAG laser with $1064 \mathrm{~nm}$ wavelength, a pulse duration of $35 \mathrm{ps}$ and $10 \mathrm{~Hz}$ repetition rate was focused onto the powder sample. The SHG behavior of KPDLiB was evidenced from the green light emission which was detected by a photomultiplier tube (PMT) coupled with a filter and converted into voltage output at a storage oscilloscope (CRO). The 


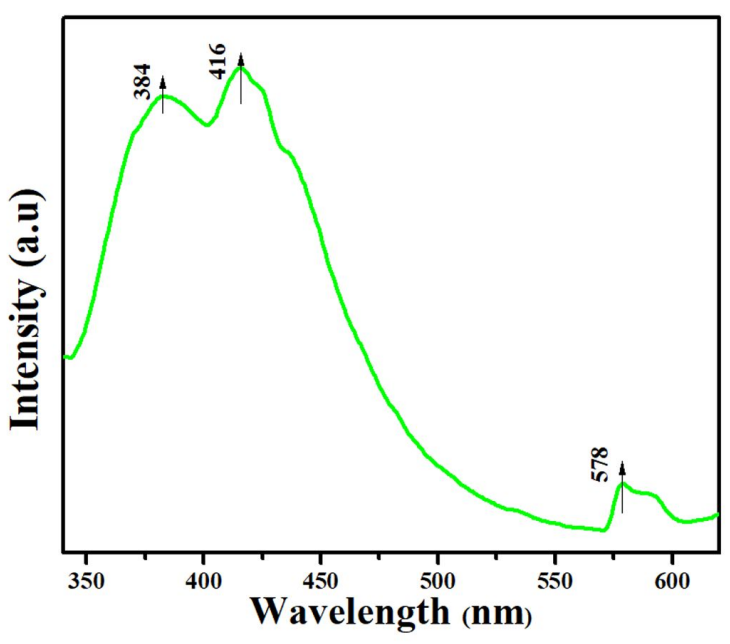

Fig. 5. PL spectrum of KPDLiB crystal.

SHG efficiency of KPDLiB was found to be comparable with that of KDP and urea. Input and output power of the laser light passed and emitted by the KPDLiB, KDP and urea are shown in Table 2. In order to study the phase matching property, the microcrystalline powder of KPDLiB was graded by standard sieves of sizes from $25 \mu \mathrm{m}$ to $210 \mu \mathrm{m}$ and then filled into separate microcapillary tubes. Fig. 6 exhibits the variation between the SHG output intensity with respect to particle sizes. The SHG output increases with increase in particle size and remains effectively stable at particle size bigger than coherence length, which confirms the phase matching ability of the grown KPDLiB and confirms the suitability of KPDLiB for frequency conversion applications.

Table 2. Comparison of SHG efficiency.

\begin{tabular}{cccc}
\hline $\begin{array}{c}\text { Input } \\
\text { power } \\
\text { [Joule] }\end{array}$ & $\begin{array}{c}\text { KPDLiB } \\
{[\mathrm{mV}]}\end{array}$ & $\begin{array}{c}\text { (Reference) } \\
\text { KDP } \\
{[\mathrm{mV}]}\end{array}$ & $\begin{array}{c}\text { (Reference) } \\
\text { Urea } \\
{[\mathrm{mV}]}\end{array}$ \\
\hline \hline 0.68 & 7.62 & 8.8 & 8.9 \\
\hline
\end{tabular}

\section{Conclusion}

Potassium phthalate di lithium borate (KPDLiB) compound was synthesized using potassium hydrogen phthalate, boric acid, lithium

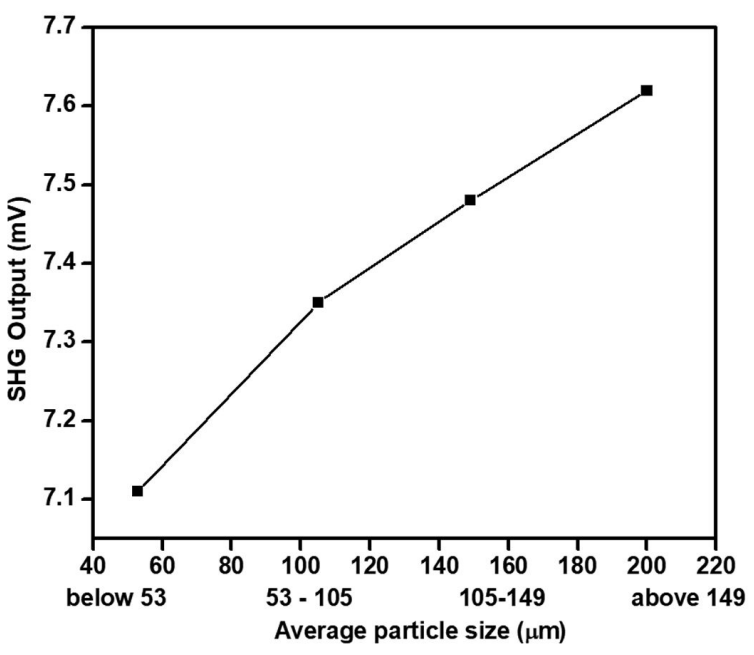

Fig. 6. The plot between average particle size and the second harmonic output values.

carbonate in equimolar ratio and the seed crystals were grown from the synthesized compound by slow evaporation technique. The bulk single crystal of KPDLiB with good morphology was grown at room temperature by slow evaporation technique. Powder X-ray diffraction analysis confirmed the crystalline nature of the grown crystal, and the structure of the grown crystal was found to belong to an orthorhombic system with space group $\mathrm{P} 2{ }_{1} 2_{1} 2_{1}$. The FT-IR spectrum recorded for the grown sample confirmed the presence of various functional groups in the grown crystal. The linear optical study revealed the good transparency of the grown crystal in a wide wavelength range which makes the crystal suitable for various electro-optic applications. The KPDLiB single crystal second harmonic generation [SHG] efficiency was confirmed by the green emission.

\section{References}

[1] Zyss J. (Ed.), Chem. Phys., (1999), 243.

[2] Karn A S. P. (Ed.), J. Phys. Chem. A, (2000), 104.

[3] Kanis D. R., Ratner M.A., Marks T.S., Chem. Rev. 94 (1994), 195.

[4] Dalal J., Sinha N., Kumar B., Opt. Mater., 37 (2014), 457.

[5] LiU G., LiU J., Zheng X., LiU Y., YUAN D., Zhang X., GaO Z., TAO X., CrystEngComm, 17 (2015), 2569.

[6] Izatt R.M., Bradshaw J.S., Dalley N.K., Chem. Rev., 91 (1981), 137. 
[7] George J., Premachandran S.K., J. Phys. D, 14 (1981), 1277.

[8] Sawyer G.A., Bearden A. J., Henins I., Jahoda F. C., Ribe F. I., Phys. Rev., 131 (1963), 1891.

[9] Miniewicz A., Mol. Cryst. Liq. Cryst., 229 (1993), 13.

[10] Chen C.T., Wu B.C., Jiang A.D., You G.M., Sci. Sin. B 28 (1983), 235.

[11] Chen C.T., Wu Y.C., Jiang A.D., Wu B.C., You G.M., Li R.K., J. Op.t Soc. Am. B, 6 (1989), 616.

[12] Timpanaro S., Sassella A., Barghesi A.Z., Por W., Fontaine P., Goldmann M., Adv. Matter., 13 (2001), 127.

[13] Chithambaram V., Jerome Das S., Arivudai NAMBI R., KRISHNAN S., Laser Technol., 43 (2011), 1229.

[14] Sivakumar B., Gokul Raj S., Ramesh Kumar G., Mohan R., J. Crystall. Proc. Technol., 2 (2012), 130.
[15] Sajan D., Vijayan N., Safakath K., Reji P., HuBERT JOE I., J Phys Chem A, 115 (2011), 8216.

[16] Senthil Pandian M., Balamurugan N., BhagavanNARAYANA G., RAMASAMY P., J. Crystal Growth, 310 (2008), 4143.

[17] Aleksandrovsky A.S., Krylov A.S., Malakhovskil A.V., POTSEluyko A.M., ZAItsev A.I., Zamkov A.V., J. Phys. Chem. Solids, 66 (2005), 75.

[18] ReICHMAn J., Handbook of Optical Filters for Fluorescence Microscopy, Chroma Technology, Brattleboro, 2010.

Received 2017-02-17 Accepted 2019-04-23 